\title{
Problem-Based Learning in Astrophysics
}

\author{
Derek Raine, \\ Department of Physics and Astronomy, University of Leicester, Leicester, LE1 7RH \\ and \\ James Collett, \\ Department of Physical Sciences, University of Hertfordshire, Hatfield, AL10 9AB \\ e-mail jdr@le.ac.uk; collett@star.herts.ac.uk
}

\begin{abstract}
Problem-based learning can be integrated into the curriculum in many different ways. We compare three examples of problem-based learning in undergraduate astrophysics programmes, and discuss the strengths and weaknesses of the various approaches.
\end{abstract}

\section{Introduction}

The traditional approach to teaching, as ancient as formal teaching itself, involves the directed flow of information from teacher as sage to student as receptacle. How effective this transmission has been can then be tested by posing various exercises to the student. Too often, however, what are actually tested are the student's patternmatching abilities. Too often, also, what appears as excellent teaching actually turns out to be facilitated pattern-matching. On the other hand, the teaching of processes (piano playing, brain surgery, home decorating) cannot be accomplished in this way. For this reason, it is hived off from cognitive education and, in the UK at least, is often treated as a less noble calling. In fact, knowledge, at the lowest level of Bloom's taxonomy [1], is of little use outside academia (and possibly TV quiz shows). The original criticism of education along these lines probably dates back to Dewey [2]. As far as any practical implication for higher education is concerned, it was realized first in medical education that in order to train medical students to solve medical problems they must be given this experience. This led to the ideas of problem-based learning (PBL), the essential features of which can be briefly summarized as students working in groups to identify and acquire the knowledge required to solve realistic problems [3]. Since its introduction in the sixties PBL has attracted a strong and ardent following.

In principle, problem-based learning translates easily to the physical sciences, where it is undoubtedly easier to understand the solution if you understand the problem. Anyone who is not convinced of this should look at a mathematical proof in an area with which they are not familiar. Every line will follow logically from the preceding lines, but it is nevertheless impossible to follow the argument intelligently without knowing what it really is that is to be proved. There are nevertheless genuine barriers to the introduction of PBL in physical science. 
Done unintelligently (or negligently) a PBL class becomes a form of teacher-free learning (a convenient time to catch up with one's knitting, as someone once put it to us). Of course, we cannot expect students to 'learn everything for themselves'. In particular, we believe that a repertoire of learnt model solutions to model problems ('proofs'), which are presented to the student, is a vital component of understanding. The fact that one appreciates these proofs differently and more deeply throughout a career is not a negation of their initial worth or of the value of committing them to memory. Nor should we forget that, especially in astronomy, students love learning pure facts, especially ones that do not require much mental processing. The main impediment to PBL in physical science in general, and in astronomy in particular, is the un-worldly nature of the subject. On the face of it there would appear to be very few 'real-world' problems in the subject, and the problems that do crop up in everyday conversation where did the universe come from? what is life?-are not exactly entry points for even your average Nobel laureate. It is the purpose of this paper to explore some ways around this and to look at the lessons to be learnt from programmes to implement these at the universities of Hertfordshire and Leicester in the UK.

To set the scene, the two university physics departments run degree programmes in physics with astrophysics for about thirty students a year and at Leicester there is also a mathematics with astronomy programme that attracts an initial entry of about ten students. The PBL projects described here were run for students at Hertfordshire at levels 0 (pre-entry) 1 and 2, and at Leicester for a class of four second year mathematics students. These very different models enable us to gain some significant insights into how PBL does and does not work (compare [4]). In the next two sections we shall describe the programmes and in the concluding section draw what lessons can be learnt. It is not our purpose here to convince the reader that PBL is the best way of teaching astrophysics to all groups of students. Rather we shall assume that the reader is interested in the potential of PBL and explore some ways of achieving these.

\section{Examples of PBL at Hertfordshire}

\subsection{Estimating Physical Quantities}

Students needs to learn to make sound estimates of physical quantities, to develop a sense of how much time to devote to estimation and to have an idea of an acceptable level of tolerance in an estimate. To develop these three skills, we use a dramatised scenario that places students in a time-critical environment in which group working is vital.

The session compresses events that might occur over a twenty-four hour period in real life into a session lasting a few hours. In studying astrophysics, students can get a sense that the long natural timescales (e.g. the lifetimes of stars) make the acquisition and interpretation of scientific knowledge, a measured and unhurried occupation. We focus on events that counter this notion. We imagine that a comet has been tidally broken up 
by the Sun and that the resultant rocky 'buck-shot' is on collision course with the Earth. The class (of around 30 students) is given a collective primary briefing and divided into groups with four or five students in each.

The students learn of events in a way that would mirror a real event of this kind. The first fragments, in the form of diffuse dust clouds, cause dramatic sunsets and unusual degrees of rainfall. The students are given mock newspaper stories describing these events. Further information release is staged, the students having constant access to a 'library' of resource materials from which they can request items. We ask them to imagine themselves members of a national task force, their job being to assimilate and interpret the data presented to them as best they can and report back at fixed intervals in a 'press conference'. We stress that in a real situation, they would be pressed for comment, so they must work toward having some answers, and providing a reasoned commentary, at the expense of over-elaborate analysis. Later information comes in different forms; dramatised TV news broadcasts from across the world are the principal source. The students have to construct a master chronology from the reports they receive from different time zones. This has proved a useful exercise and we recommend to the groups at the outset that one member of the team should act as 'time-keeper'.

The students are given written transcripts of the satellite broadcasts and further information is also relayed in the form of fax and e-mail messages, seismograms and press releases. At each stage of the exercise the students receive typically four or five questions, with three or four stages in total. This allows the students to improve their analytical efficiency even as the session unfolds. Questions are both qualitative (e.g. Could the tsunami wave off the Japanese coast have been triggered by volcanic activity?) and quantitative (e.g. What size of object caused the crater in Portugal?). The students are encouraged, not only to frame answers to the questions, but to anticipate the unfolding sequence of events. In practice, they have little time to do the latter, a reflection no doubt of what would happen in a real event of this kind!

It is important that the onus is on the group to find efficient ways to assimilate and organize the different data they receive. One common problem is that the groups fail to do this internally, so that before reporting back they do not allow themselves time to assess their knowledge base as a team. They would clearly benefit from smaller scale practice of the techniques in advance. One preparatory exercise we have used involves the Tunguska event, the explosion of a rocky body in the atmosphere over Siberia in 1908. We ask the students to figure out the visibility of the object in the hours and days before the impact and the speed of dispersal of dust across the globe. As an example, we point out to students that eyewitness accounts can be unreliable. When a Japanese fisherman has to assess the brightness of a bolide's trail, the likely visual references, the Sun and Moon, differ in brightness by five orders of magnitude.

Our experience was that the news broadcasts worked well, the students proving competent in picking out relevant details aurally without recourse to the transcript. The 
most significant problem that students encounter is translating this information into the kind of 'back of the envelope' numbers required for the press conferences. Once the students recognize their need for this new skill, some assistance is given in order to maintain the momentum of the exercise.

One problem can be students who make little contribution early in the session, who lose interest and with it the confidence of their team. Individuals can also dominate within a group, perhaps in a very particular skill such as rapid mental calculation. One way to counter these difficulties is to have the staged questions sub-divided into categories of skills e.g. estimation, data analysis, graphical and image interpretation. At each press conference, the members of a group can be forced to swap roles and tackle a different type of problem. However, it is important that the group dynamic is not lost; a group should brainstorm first as a team before splitting the assignment. The fact that a group has to report back as a team ensures that they should share their results with each other before 'going public'. Although we have not done this, it might be interesting for the press conference to target individuals for answers independently of their role in the group. The group would then be forced to educate each other to approximately the same level of proficiency and it would create a sense of group ownership of ideas.

The construction of the scenario in an extended exercise is very important, and the ending must not disappoint. We have one final cometary fragment of a size that would lead to global catastrophe, although a near miss might prove a more optimistic ending. At the end of the session, the students are taken through the day in chronological sequence as a class. A fully worked written account of the events is given to each student. They can then compare their estimates against the truth and reflect on techniques of estimation - a very strong learning experience. They can also see the context of the catastrophic events of a single day in the broader setting of human history: the comet was disturbed from the Oort cloud a few million years before it was detected on Earth.

Our experience is that students find the exercise interesting but tiring. There may be merit in less ambitious schemes, where the scope of the questions is narrower and particular techniques are developed, although one merit of this session is the testing of synoptic skills.

We have not yet run this session as an assessed exercise because it is still experimental in form. The focus of assessment would clearly fall on the accuracy and clarity of the commentary that was offered by each team, but the contributions of individual group members should also be addressed. The press conference gives a good occasion to assess communication skills. There are also opportunities for students to reflect on how they might have performed better.

The scientific content of this session is high; good practice is possible in problems of dynamics, magnitudes and the use of logarithms, trigonometry and angular measure 
and other core areas of the curriculum. As a shock therapy, to illustrate that real problems are challenging, and can be attacked in different ways with elementary mathematics, it works well. A revised and expanded version of this project is being developed for schools and colleges under a PPARC award.

\subsection{Alien environments}

One of the challenges in teaching astrophysics is to instill in students a sense of place - a physical 'feel' for unfamiliar environments as diverse as, for instance, planetary surfaces, stellar atmospheres and high-energy particle jets. In a course on solar system physics, we have used problem-based learning to persuade students to worry about the detailed features of one such environment.

Their brief is to design a robot mission to Europa, one of the moons of Jupiter. Europa is chosen because of the current interest in this moon as a possible abode for primitive life. This discussion has been motivated by circumstantial evidence for extensive oceans on Europa. The oceans sit beneath the superficial ice crust, and are warmed from below by heat released in the tidal stretching of the moon's interior. The students have many aspects to consider in their mission design and we recommend they follow professional practice in dividing labour and responsibility by creating mission specialists. The brief is that they must create a mission plan that will capture the imagination of private sponsors. One reason for doing this is the notional plans that space agencies have for Europa. It is clear to the students that external and independent sponsors are unlikely to be attracted by a mission that may be funded and executed by a space agency. Accordingly, we give weight in the assessment to the originality of their concept and the entrepreneurial flair with which it is presented.

The students are given limited additional guidelines although we emphasise that the mission would be unlikely to receive funding if the task of searching for life were sketchily addressed. The students have to balance the technical complexity of their mission design with its chances of satisfying this objective. Advanced submarine designs are criticised if no thought has been given to communication with the surface and orbiter relay, or to redundancy in the event of partial systems failure. Credit is given to ideas that exploit the local environment and suggestions for contingency measures if the environment proves slightly different to what was expected. Students are encouraged to suggest where new materials need to be developed or might exploit the environment (e.g. high temperature superconductors). Any realistic impetus that their plans might give to industrial development, with a wider significance than the mission, is commended.

We created a notional 'foundation' that would send a panel of representatives to sit through presentations and mark written submissions. The panel consisted of scientists and a model designer from the Faculty of Art and Design, who has experience on national television science programmes. There are obvious opportunities for further interdisciplinary collaboration (e.g. with engineers, biologists, geologists, environmental 
and material scientists, animators and model design students joining the teams). The problem here is that most university courses are not designed with assessment in such shared projects in mind. The irony is that this close simulacrum of real professional practice is exactly what should be practised and assessed.

\section{A Leicester experience}

We have deliberately chosen this only partially successful example as a contrast from which we can learn something. In the interests of ethical behaviour it should be said that we did not set out deliberately to implement a less successful model, but to see how well we could use PBL within some given external constraints. The examination results for this group of students were certainly no worse, and probably somewhat better than previous comparable groups on this programme, and they were therefore not disadvantaged by the experiment. We should add that we have numerous other more successful examples that would however be of less interest for this paper.

Briefly, the second year students taking mathematics with astronomy would follow the same course of thirty-six lectures over a year and take the same three-hour examination as our physics students. The lectures cover positional astronomy, planets, stars, galaxies and cosmology in a quantitative manner, the first year course having provided a nonmathematical introduction to the same subjects. In the past, some tutorial support has been provided in various forms, but the results for the mathematics students in recent years have been disappointing. Clearly the students were not really engaging with the material. In view of this we decided to change the course for the mathematicians to study the effects of PBL, in the hope that the problems would be interesting enough, and the lack of given material threatening enough, to promote engagement.

In order to provide a valid test we kept the same written examination as in previous years, the examination material being known to the course designers, as in all other cases where the lecturers set the examinations. This constrained the development of the course. In fact, as we went along, the pressure of time dictated by the need to prepare for the examination forced us increasingly to act as tutors and not facilitators. This illustrates a basic and well-known feature of PBL: the amount of material that can be covered is less than in a conventional lecture course. Attendance at the lectures was made voluntary, and, except for positional astronomy, the students chose not to attend. There were five problems: on the discovery of the Kuiper belt objects, particularly Varuna [5], on the possible inhabited planets round a star of a given spectrum, on the radii of stellar clusters in the solar neighbourhood, on the masses of galaxy clusters and on cosmology. With the exception of the positional astronomy all the material required to answer the questions was taken from a single astronomy textbook.

We encountered all manner of problems from the start. With only one group it is difficult to impose deadlines. Conversely, having only one PBL course, the group initially found too many other demands on their time to meet together out of the scheduled one or two hours per week, or to do the research. It took many weeks to get them 
organized, not helped by their absence of experience in group work and their perception of the difficulty of the first problem. As we went through the course the problems became less imaginative and more like end-of-chapter exercises, and the students came to like them much more!

In addition to the examination we marked solutions submitted individually, but we did not assess the group performance as such. Nevertheless, we did perceive some development of their ability to function as a group, particularly to swap felicity at calculation for insight into physics. On the other hand, as usual, students use the marking scheme to judge when they have done enough.

The experience provided us with several major insights of which two are worth recording. The first was the extent to which these students thought of physics as purely and simply a matter of knowing 'the formula'. (Talking about blackbody radiation for the umpteenth attempt, one student victoriously volunteered a formula: 'sigma, $T$ to the fourth, W over $m$ squared'.) Extended mathematical arguments for these students were something you 'did in maths', and, because they saw no logical structure in physics, proofs were something they believed had to be learnt by rote. The second insight was the difficulty that the students had with reading a technical text: they seemed to be adept only at pattern matching formulae. This would not have been revealed by a traditional lecture course, and once uncovered, we were able to provide assistance.

Common to both the implementations was the shock factor that the work required more than pattern matching of formulae. This encouraged some degree of inquisitiveness and understanding and some appreciation of the wonder of astrophysics.

\section{Discussion}

An essential element of PBL is group work, but the group aspects cannot be artificially imposed. The problems have to be chosen so that they are naturally approached by group work. The Hertfordshire examples embed this quite naturally. Conversely, if something cannot be naturally framed in a group context, other teaching methods might be more appropriate. PBL is not an exclusive methodology.

On the other hand, group work itself is not necessarily PBL. In two of the three examples presented here the problems were rather closed, the range of what would count as a correct answer being rather limited. The question as to whether this is really then PBL is not merely a semantic point. It is precisely the fact that a true PBL problem can be answered at many levels that enables students, with appropriate facilitation, to build constructively, step by step, on prior-learning.

A useful feature of group learning is the competitive element. This can be quite surprising and invigorating when it works and depressing when it does not. The PBL 
approach can be undermined by a collective determination that the facilitator should turn tutor. To this end it can help if the whole PBL session is supervised in a single class, but this approach does not readily support the strategic acquisition of new knowledge over an extended timescale.

An important consideration throughout is the extent to which group work is developed prior to PBL. We have seen in all our examples that this would have helped. In the literature one can find extensive discussion of the extent to which team roles should be made explicit. It is clear from our experiences that at least some prior discussion of team roles enables students to reflect on their approach to teamwork and to realize that they are supposed to be working as a team.

It is a commonplace to say that the most important factor in learning is prior knowledge. PBL is supposed to value all prior knowledge as a valid contribution to the group effort. It is however very easy to see how didactic instruction (telling students what you want them to know) is more efficient than PBL if the problems do not mesh with the prior knowledge of the group, because it is then difficult for the students to understand the problems well enough to get started. This makes it impossible to get through a set syllabus in a given time unless you are sure of your starting point. But, taking the optimistic view, this is the strength of PBL: the syllabus can be reduced so that there is time for knowledge to be properly embedded.

It is obvious therefore that the freedom to define a syllabus is an important factor in the viability of PBL. A freedom to define the overall learning environment is another. For students coming from the mathematics department, physics is already an alien environment. There should therefore be no expectation that the course be taught like a mathematics course. Even so, our experience shows how the 'long-thin' approach to PBL (for example, a single module spread over a semester) is more difficult to bring off than the 'short-fat' one (a period devoted entirely to one PBL activity). Of course, one could not make a programme out of one-day PBL classes alone, which means that in practice a compromise is needed. If $\mathrm{PBL}$ is to be successful it has to be designed into the programme, not carved out of it.

In the longer term the Universities of Leicester and Hertfordshire are part of a HEfCE funded UK project (including Reading and Sheffield) to investigate ways of introducing PBL into the teaching of physics and astrophysics (project LeAP). This will enable us to investigate more fully student and staff attitudes and the costs and benefits of the various approaches.

\section{References}

[1] Bloom, B.S. (Ed.) (1956) Taxonomy of educational objectives: The classification of educational goals: Handbook I, cognitive domain. New York ; Toronto: Longmans, Green. 
[2] Dewey J (1963) Experience and Education (New York: Collier)

[3] Barrows H S (2000) Problem-Based Learning Applied to Medical Education (Southern Illinois University School of Medicine, Springfield, III.)

[4] Savin-Baden M. (2000) Problem-based Learning in Higher Education: Untold Stories (UK: SRHE and Open University Press)

[5] Jewitt D, Aussel H \& Evans A., (2001) The size and albedo of the Kuiper-belt object (20000) Varuna Nature 411, 446 